\title{
Use of the PLIF and LIP methods for definition of non-stationary temperature fields of water droplets
}

\author{
Roman S. Volkov ${ }^{*}$, Maria M. Osmolovskaya, and Yaroslav S. Solomatin \\ National Research Tomsk Polytechnic University, 634050 Tomsk, Russia
}

\begin{abstract}
This study illustrates the results of experiments to determine the unsteady and non-uniform temperature fields of evaporating water droplets. It is shown that during the warming up of the droplet inside it an inhomogeneous temperature field is formed. In the droplet trace, formation of a region with lower (relative to the gas flow) temperatures of the vapor-gas mixture is observed. The experiments were carried out for droplets with initial radii of $1-2 \mathrm{~mm}$. The speed of the air flow was $0.5-5$ $\mathrm{m} / \mathrm{s}$. The temperatures of the gaseous medium varied in the range of $20-$ $600{ }^{\circ} \mathrm{C}$. During the research, modern panoramic methods of non-contact flow diagnostics such as "Planar Laser Induced Fluorescence" and "Laser Induced Phosphorescence" (LIP) were used.
\end{abstract}

\section{Introduction}

Modern models of heat and mass transfer describing the heating and evaporation of liquid droplets in a gaseous medium are formulated on the basis of long-established hypotheses and representations by Spalding D.B. [1], Fuchs N.A. [2], Ranz W.E. and Marshall W.R. [3]. The development of these concepts over the last 30 years has allowed us to obtain a group of approximations which illustrate the corresponding dependences of Nusselt numbers on the Reynolds and Prandtl numbers. These expressions are traditionally used in the simulation of convective-conductive heat and mass transfer processes and phase transformations at the liquid-gas interface. The results of the studies [4] showed that in the region of moderate (up to $600 \mathrm{~K}$ ) gas temperatures, the use of the expression group $\mathrm{Nu}=\mathrm{f}$ $(\mathrm{Pr}, \mathrm{Re})$ makes it possible to obtain a satisfactory agreement of the theoretical values to the experimental data (the differences do not exceed 10-15\%). At temperatures above $500{ }^{\circ} \mathrm{C}$, the theoretical and experimental values of the evaporation rates of water droplets differ by several times [4]. A hypothesis [4] has been advanced that at high temperatures of a gaseous medium, the non-stationary processes of formation of a substantially inhomogeneous temperature field of evaporating water droplets can play a decisive role. With intense heating, the temperature distribution in the droplet can be substantially

\footnotetext{
*Corresponding author: romanvolkov@tpu.ru
} 
nonuniform and nonstationary. Unfortunately, up to the present time, adequate experimental methods for determining the temperature distributions inside the droplet have not been developed.

Contactless optical methods for diagnosing thermophysical parameters (such as Particle Image Velocimetry (PIV) [2], Particle Tracking Velocimetry (PTV) [3], Planar Laser Induced Fluorescence (PLIF) [4] and others) have been actively developing over the past few years. To solve the previously identified problems and determine the temperature fields of the evaporating water droplets, it is expedient to use the methods of PLIF [4] and LIP [5, 6], since it allows the recording of the temperature of liquid and gas media with high sampling in time and space with high sampling.

The purpose of this work is to determine the features of heating and evaporation of water droplets during high-temperature heating in a gaseous medium using non-contact panoramic optical methods "Planar Laser Induced Fluorescence" (PLIF) and "Laser Induced Phosphorescence" (LIP).

\section{Experimental setup and procedure}

Fig. 1 show the experimental setup used in carrying out experimental researches.

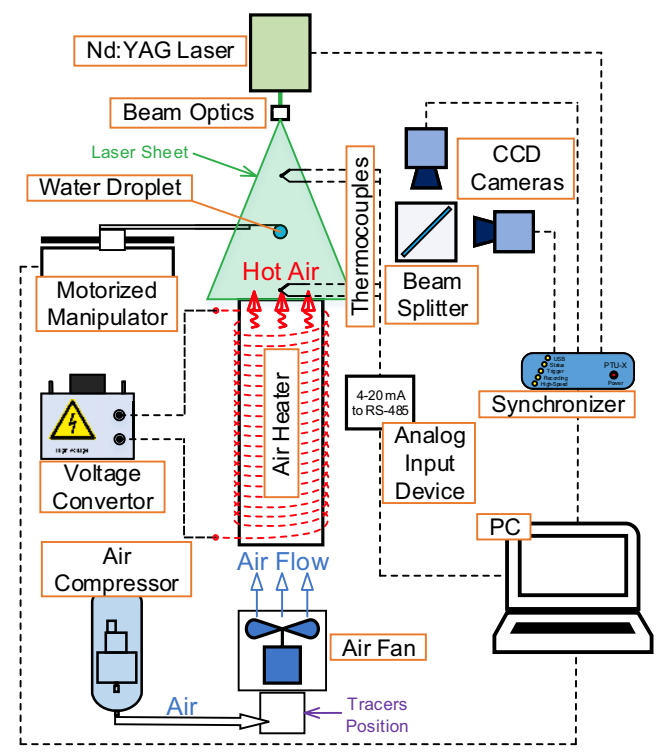

Fig.1. A scheme of experimental setup.

The main element of the setup (see Fig. 1) was the heating element. The heating element consisted of a hollow ceramic cylinder (inner and outer diameters $60 \mathrm{~mm}$ and 52 $\mathrm{mm}$ ) $1 \mathrm{~m}$ high, the external circuit of which is provided with 6 independent windings (of nichrome wire $0.3 \mathrm{~mm}$ in diameter) per 1 winding for every $0.2 \mathrm{~m}$ of the ceramic pipe. The total maximum power consumption of nichrome spirals was $9 \mathrm{~kW}$. The airflow in experiments was pumped by a fan connected to the lower inlet of the heater (ceramic tube). The speed of the air flow was $0.5-5 \mathrm{~m} / \mathrm{s}$. The air flow rate was monitored using the PIV 
method [2]. The temperature of the air stream $\left(T_{\mathrm{a}}\right)$ at the outlet of the heater (the upper part of the ceramic tube) was regulated in the range $20-350{ }^{\circ} \mathrm{C}$ by changing the voltage from 50 to $250 \mathrm{~V}$ on the nichrome windings. Two lowering autotransformers were used. Type-K thermocouples (temperature measurement range $223-1473 \mathrm{~K}$, accuracy $\pm 3 \mathrm{~K}$, heat retention no more than $0.1 \mathrm{~s}$ ) were used to measure temperature of hot air flow (see Fig. 1).

To generate water droplets of fixed sizes, the Finnpipette Novus electronic dispenser $\left(0.1 \mu 1\right.$ volume variation step) was used. The initial volume $\left(V_{\mathrm{d}}\right)$ of the generated droplets ranged from $5 \mu \mathrm{l}$ to $30 \mu \mathrm{l}$. This corresponds to a change in the droplet radii in the range $R_{\mathrm{d}}=1-2 \mathrm{~mm}$.

The PLIF [4] and LIP [5, 6] method was used to determine the instantaneous temperature distributions inside the water droplet and in the trace of the evaporating droplet. The schemes of the methods are shown in Fig. 2.
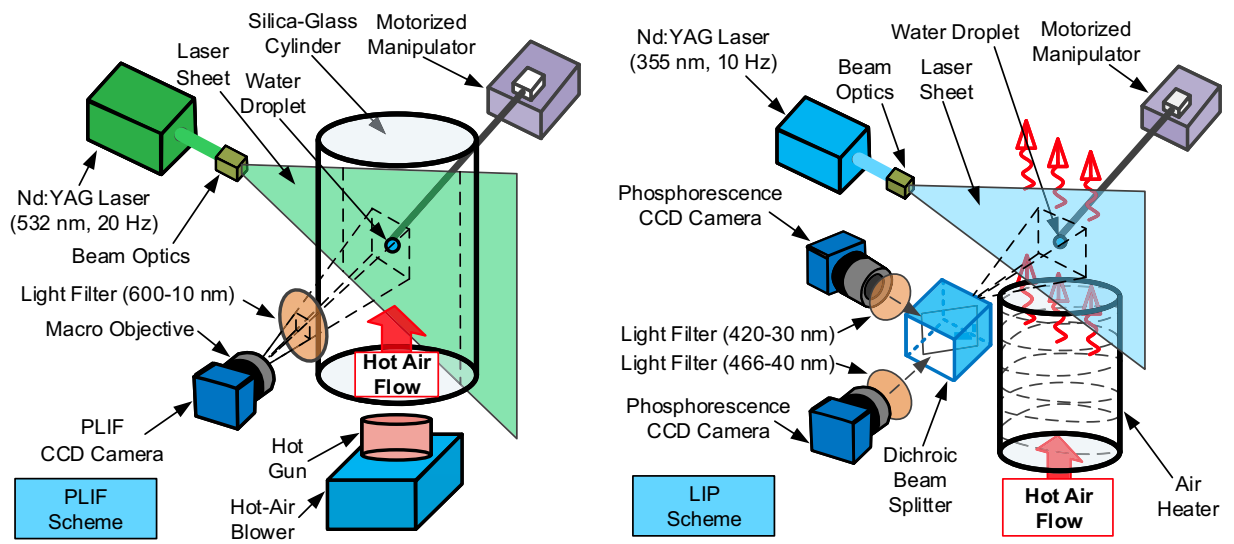

Fig. 2 The schemes of the PLIF and LIP methods.

To illuminate the water droplet in the experiments used solid-state pulsed Nd: YAG Quantel lasers (wavelength $532 \mathrm{~nm}$ and $455 \mathrm{~nm}$ ). To create a laser sheet laser optics (based on cylindrical lenses) with an opening angle of $8^{\circ}$ was used. Images of water droplets were recorded by CCD ImperX IGV-B2020M and Imager M-lite $2 \mathrm{M}$ cameras.

The experiments with the use of PLIF and LIP (Fig.4) were performed in two stages: the first was the temperature calibration of the system (in the absence of a drop of water in the registration area), the second - the recording of the temperature fields of the evaporating droplet [4-6].

As specialized tracers for flow in the LIP method, specialized phosphor particles of $\mathrm{BaMgAl}_{10} \mathrm{O}_{17}$ :Eu powder (BAM: Eu) $[5,6]$ were used, the particle sizes were 6-8 $\mu \mathrm{m}$. To avoid clumping of particles in the flow, they were first placed in a muffle furnace (at 100 ${ }^{\circ} \mathrm{C}$ ) for $120 \mathrm{~min}$ (in accordance with the recommendations and conclusions [5, 6]). As a fluorophore in the PLIF experiments, Rhodamine B was used [4]. The selected fluorophore is sufficiently stable under the action of a laser; moreover, the intensity of the fluorescent emission of a given dye has a pronounced temperature dependence: the decrease in fluorescence intensity is about $2 \%$ per $1{ }^{\circ} \mathrm{C}$ in the range $10-70{ }^{\circ} \mathrm{C}$. The errors in measuring the temperature using the PLIF method did not exceed $2{ }^{\circ} \mathrm{C}$, the error of the LIP method was about $25^{\circ} \mathrm{C}$. 


\section{Results and discussions}

Fig. 3 shows temperature field inside the droplet of water obtained using the PLIF method. PLIF images allow us to observe non-steady and non-homogeneous temperature fields of droplets throughout the long heating intervals achieving several tens of seconds. A nonsteady behavior of the temperature field becomes obvious when comparing $T_{\mathrm{d}}$ values in identical droplet sections during various time intervals. These values quickly change with the increase in heating time. Thus, Fis. 3(a) illustrate the difference of $30-40{ }^{\circ} \mathrm{C}$ in the identical cross-sections, while in Fig. 6(b) this difference is not over $10^{\circ} \mathrm{C}$. From this we conclude that the increase in heating time results in practically homogeneous temperature field. Certain constant values of $T_{\mathrm{d}}$ to which the droplets heat up before their complete evaporation, are observed in each experiment. The droplet temperature field is strongly affected by the direction of the hot airflow. The higher $T_{\mathrm{a}}$ and $U_{\mathrm{a}}$ values the higher temperature rise on the droplet frontal surface unlike its back and lateral surfaces (Fig. 3).

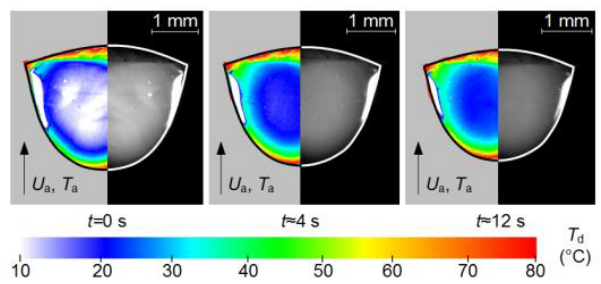

(a)

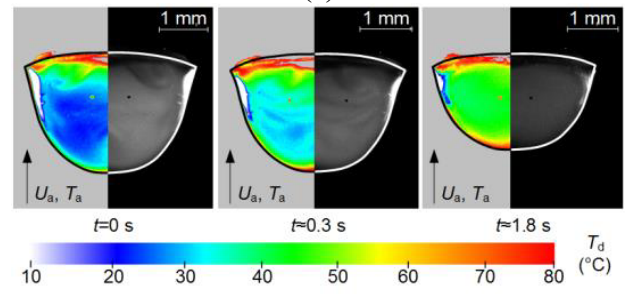

(b)

Fig. 3. Time history for droplet temperature field (PLIF method) at different heating conditions $\left(U_{\mathrm{a}} \approx 3\right.$ $\mathrm{m} / \mathrm{s}, R_{\mathrm{d}} \approx 1.53 \mathrm{~mm}$ ): (a) $-T_{\mathrm{a}} \approx 100{ }^{\circ} \mathrm{C}$; (b) $-T_{\mathrm{a}} \approx 500{ }^{\circ} \mathrm{C}$.

Fig. 4 shows temperature field outside the evaporating droplet of water obtained using the LIP method. The recorded temperature trace, its longitudinal and transverse dimensions (Fig. 4) is visually shown. In contrast to the thermocouple measurements [10] in this paper, it was possible to determine the essentially non-stationary nature of the formation of a temperature field around evaporating water droplets. In particular, an intensively changing temperature trace is recorded both in the longitudinal and transverse directions relative to the incoming flow of heated air. With an increase in the heating time and a decrease in the droplet size, the latter is heated to a practically homogeneous temperature field (Fig. 4). As a consequence, heat transfer processes on the droplet surface stabilize somewhat, evaporation rates acquire maximum values. Under such conditions, the cooling of the flowing gases is due to intensive evaporation due to the high heat of vaporization. Only when a certain small value of the radius of the evaporating droplet reaches its temperature trace does it begin to decrease sharply. In Fig. 4, one can see a sharp decrease in longitudinal and transverse dimensions (when $t \approx 35 \mathrm{~s}$ ). 


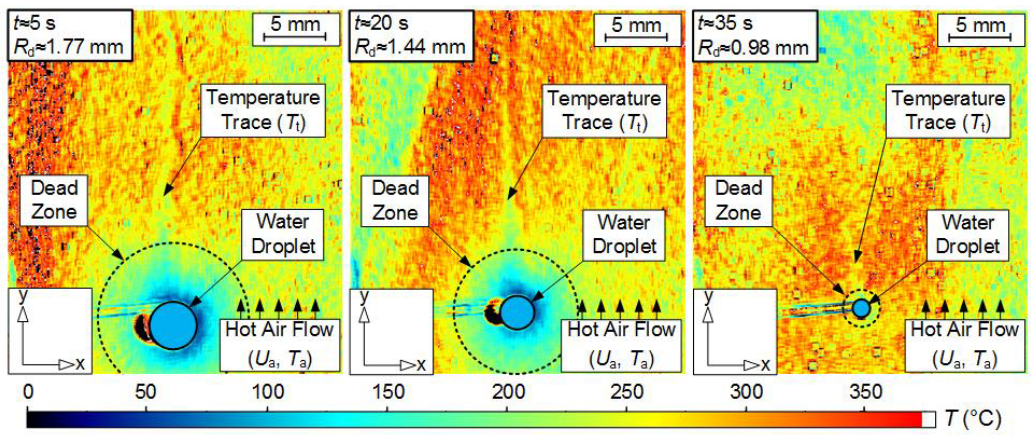

Fig. 4. Non-stationary and significantly non-uniform fields of temperature (LIP method) of a gas stream at a water drop flowing $\left(U_{\mathrm{a}} \approx 3 \mathrm{~m} / \mathrm{s}, R_{\mathrm{d}} \approx 1.81 \mathrm{~mm}\right)$.

The obtained generalized results of the conducted experiments are the fundamental basis for the development of a number of promising technologies, for example, fire extinguishing, thermal cleaning, etc. In particular, with their application, it is possible to develop perspective physical and mathematical models that allow predicting the required heat exchange conditions for a drip aerosol with a hot air flow.

\section{Conclusion}

In the course of the research, the possibility of applying the PLIF and LIP methods for the diagnostics of the temperature distribution of an evaporating droplet was demonstrated. The performed experiments showed the presence of a substantially non-stationary temperature field both inside the drop itself and in its trace. It is established that the temperature drop inside the water droplet can reach up to $50{ }^{\circ} \mathrm{C}$. The decrease in temperature in the droplet trace due to its evaporation can reach values of $60-80{ }^{\circ} \mathrm{C}$ (in comparison with the gas flow temperature). In this case, the temperature trace of the droplet is retained throughout the evaporation process. The results obtained can be used to develop ideas of high-temperature evaporation of droplets, as well as the development of appropriate mathematical models

The research was supported by Foundation of President of Russian Federation (project MD-1221.2017.8).

\section{References}

1. D.B. Spalding, Some fundamentals of combustion, Butterworth's, London (1955)

2. N.A. Fuchs, Evaporation and Droplet growth in Gaseous Media, Pergamon Press, London (1959)

3. W.E. Ranz, W.R. Marshall, Chem. Eng. Prog. 48 141, 173 (1952)

4. O.V. Vysokomornaya, G.V. Kuznetsov, P.A. Strizhak, J. Eng. Phys. Thermophys. 89 $141(2016)$

5. F. Yan, A. Rinoshika, Exp. Therm. Fluid Sci. 44534 (2013)

6. D. Damiani, E. Meillot, D. Tarlet, J. Therm. Spray Technol. 23 (3) 340 (2014)

7. B. Williams, P. Ewart, X. Wang, R. Stone, H. Ma, H. Walmsley, R. Cracknell, R. Stevens, D. Richardson, H. Fu, Combust. Flame 157 (10) 1866 (2010)

8. A. Charogiannis, F. Beyrau, Exp. Fluids 541518 (2013)

9. C. Abram, B. Fond, A.L. Heyes, F. Beyrau, Appl. Phys. B. 111 (2) 155 (2013)

10. R.S. Volkov, G.V. Kuznetsov, P.A. Strizhak, I.S. Voytkov, Int. J. Multiph. Flow 91184 (2017) 